\title{
Absence-like seizures in adult rats following pilocarpine-induced status epilepticus early in life
}

B.L.C. Ferreira ${ }^{1,2}$, A.C. Valle ${ }^{1}$, E.A. Cavalheiro ${ }^{2}$ and C. Timo-laria ${ }^{1}$

\author{
'Laboratório de Neurocirurgia Funcional, Faculdade de Medicina, \\ Universidade de São Paulo, São Paulo SP, Brasil \\ ${ }^{2}$ Laboratório de Neurologia Experimental, Escola Paulista de Medicina, \\ Universidade Federal de São Paulo, São Paulo SP, Brasil
}

\section{Correspondence \\ C. Timo-laria \\ Laboratório de Neurocirurgia \\ Funcional, FM, USP \\ Av. Dr. Arnaldo, 455 \\ 01246-903 São Paulo SP \\ Brasil \\ Fax: +55-11-3062-2897 \\ E-mail: cetiaria@usp.br}

Research supported by FAPESP,

CPNq, PRONEX, HCFMUSP and FFM.

Received March 24, 2003

Accepted September 5, 2003

\begin{abstract}
Administration of pilocarpine causes epilepsy in rats if status epilepticus (SE) is induced at an early age. To determine in detail the electrophysiological patterns of the epileptogenic activity in these animals, 46 Wistar rats, 7-17 days old, were subjected to SE induced by pilocarpine and electro-oscillograms from the cortex, hippocampus, amygdala, thalamus and hypothalamus, as well as head, rostrum and vibrissa, eye, ear and forelimb movements, were recorded 120 days later. Six control animals of the same age range did not show any signs of epilepsy. In all the rats subjected to SE, iterative spike-wave complexes $(8.1 \pm 0.5 \mathrm{~Hz}$ in frequency, $18.9 \pm 9.1 \mathrm{~s}$ in duration) were recorded from the frontal cortex during absence fits. However, similar spike-wave discharges were always found also in the hippocampus and, less frequently, in the amygdala and in thalamic nuclei. Repetitive or single spikes were also detected in these same central structures. Clonic movements and single jerks were recorded from all the rats, either concomitantly with or independently of the spike-wave complexes and spikes. We conclude that rats made epileptic with pilocarpine develop absence seizures also occurring during paradoxical sleep, showing the characteristic spike-wave bursts in neocortical areas and also in the hippocampus. This is in contrast to the wellaccepted statement that one of the main characteristics of absence-like fits in the rat is that spike-wave discharges are never recorded from the hippocampal fields.
\end{abstract}

\author{
Key words \\ - Epilepsy \\ - Absence seizures \\ - Spike-wave \\ - Status epilepticus \\ - Pilocarpine
}

\section{Introduction}

Epidemiological studies indicate that human epileptic disorders frequently have their onset early in life. The highest incidence of epilepsy is found in childhood and adolescence $(1,2)$. From 1 to $8 \%$ of all epileptic patients present at least one episode of status epilepticus (SE) and a considerable number of these patients die as a consequence of SE (2-5). Mortality due to SE ranges from 2.5 to $20 \%$ in adults (5) and may reach moderately higher values of up to $25 \%$ in children (2-8). These data suggest that SE is a prominent factor in epilepsy, as also demonstrated experimentally in rats and mice (9-14). In humans, late epileptic seizures may occur years after SE (4) and it should be pointed out that 
in children and adults who die of SE, lesions have been found in several areas of the brain $(2,4,8)$.

Although epilepsy may become manifest at any age, the onset of its causing lesion does often occur shortly after birth, when the nervous system is still immature and highly susceptible to metabolic, traumatic and inherited damaging factors (3). The phases of neural maturation during which such factors exert their influence seem to be a key factor in determining the threshold for the development of any kind of epileptic seizure, either convulsive or non-convulsive (15-18).

Many manifestations of epilepsy can be studied more closely in laboratory animals than in humans, providing valuable information about this disease. Among such manifestations, SE and epileptic discharges in several areas of the brain are the most convenient to be studied in animal models, also for testing antiepileptic drugs. The pilocarpine model of epilepsy in rats is well suited to the study of several kinds of epilepsy that mimic human epileptic fits. In fact, systemic administration of pilocarpine, a cholinergic muscarinic agonist, to rats 7 to 10 days after birth causes mild behavioral manifestations, such as motor hyper- or hypoactivity, head and body tremor and scratching. When injected into 11- to 17-day-old rats, however, pilocarpine usually induces far more severe effects, such as mastication, salivation, eye blinking, jaw and limb clonic movements, rearing, and generalized convulsions. In both cases, however, the rats eventually progress to SE (18-20). The long-term consequences of SE, such as those observed in adult rats and mice $(10,11)$, are spontaneous seizures secondarily generalized and accompanied by structural damage to the brain. Chronic epilepsy thus provoked is similar in many aspects to human temporal lobe epilepsy and as such is a good model for this human disease.

Even brief episodes of SE may cause injury to neurons in several parts of the brain. Particularly vulnerable to SE caused by pilocarpine are the hippocampal interneurons (21) but granule cells in the dentate gyrus also are lesioned, exhibiting, like the interneurons, morphological features of apoptosis and necrosis $(22,23)$. It is interesting to note that reinduction of SE does not provoke greater damage to these neurons (24).

Since recording the electrical activity (electro-oscillograms) of a large number of cortical and subcortical sites in the brain is of the utmost relevance for the study of epileptic seizures, the present study was carried out to record the electro-oscillograms from several brain areas and to assess the body movements of adult rats subjected to systemic pilocarpine administration at the age of 7 to 17 days. Some unexpected manifestations of this type of epilepsy were detected by the wide span analysis of the electrical activity of the brain in these animals.

\section{Material and Methods}

Forty-six Wistar rats of either sex (16 females and 30 males), permanently kept in a temperature-controlled environment under a 12-h light-dark cycle, were injected ip with a single dose of pilocarpine $(320 \mathrm{mg} / \mathrm{kg}$ ) between 7 and 17 days after birth $(10,18)$. Six age-matched control animals were injected with $0.9 \%$ saline. The animals were subjected to implantation of electrodes 120 days after $\operatorname{SE}(10,20)$ for recording the electro-oscillograms and body movements. Preliminary studies showed that after this interval following pilocarpine administration the changes in the electrophysiological manifestations of epilepsy were stabilized.

Parallel nichrome electrodes $150 \mu \mathrm{m}$ in diameter, bent at $90^{\circ}$ at the distal $0.5 \mathrm{~mm}$ and isolated except at the end cut section (to detect potentials from a small area), were stereotaxically implanted under $50 \mathrm{mg} / \mathrm{kg}$ ip cyclo-hexylamine $\left(\right.$ Ketalar $\left.^{\circledR}\right)$ anesthesia. The electrodes were implanted over cortical areas 3 and 10 according to skull references 
$(25,26)$ that make it possible to implant the electrodes over specific cytoarchitectonic and functional (27) areas through 1-mm holes in the bone.

Similar but straight electrodes parallel and isolated except at the end cut crosssection were stereotaxically implanted into the $\mathrm{CA}_{1}$ and $\mathrm{CA}_{3}$ hippocampal fields according to the Paxinos and Watson atlas (28). In 10 rats electrodes were also implanted in the amygdala and in the ventral posterolateral thalamic and ventromedial hypothalamic nuclei. Nichrome electrodes were implanted in the trapezius muscles, in each external canthus of both eyes, in the muscle pad that moves the snout and vibrissae and in the biceps brachialis, to record head, eye, rostrum and vibrissa and forelimb movements, respectively. Not all the electro-oscillograms or all the movements were recorded in every experiment.

The electrodes were connected to the pins of a 26-pin socket, which in turn was anchored to the bone by two brass screws 1.4 $\mathrm{mm}$ in diameter and thereafter cemented to the skull with a rapidly polymerizing methacrylate. After electrode implantation the animals were kept in individual cages, with free access to food and water. The procedures for implanting the electrodes were performed with extreme care to avoid edema that usually results in unreliable recordings before one week post-surgery.

For the electro-oscillographic recording, which was performed with a 21-channel Nihon-Kohden electroencephalograph, the rat was placed in a small cubic Faraday metal cage $(0.40 \times 0.40 \times 0.40 \mathrm{~m})$ for electrical shielding, and the socket fixed to the bone was connected to the recorder input panel. Recordings, which began 2-3 days after electrode implantation, were continuously monitored for 6-8 $\mathrm{h}$ and repeated every three days for four weeks. Since it is well known that spike-wave discharges (SWD) and absence seizures are specifically blocked by certain drugs, in 6 rats ethosuximide was injected
(200 $\mathrm{mg} / \mathrm{kg}$, ip) to find out if the spike-wave complexes would be blocked.

At the end of the recording series each rat was injected with an overdose of cyclohexylamine and a small, marking electrolytic lesion was provoked at the distal tip of every subcortical electrode, intended to check its correct placement. The head was subsequently perfused with $250 \mathrm{ml}$ of $0.9 \%$ saline and with a similar amount of $10 \%$ formalin thereafter. The brain was removed and kept in formalin for seven days at $10^{\circ} \mathrm{C}$, after which $20-\mu \mathrm{m}$ frozen sections were Nisslstained, only for the purpose of identifying the sites where the electrodes had been implanted.

\section{Results}

\section{Neocortical spike-wave bursts}

SWD were recorded from cortical areas 10,3 and 17 of all experimental rats. The epileptic patterns were very similar for all 46 animals. The spike-wave complexes were bilateral and synchronous, although seldom with a similar voltage on both sides; occasionally, in the same animal, SWD started 0.1 to $1 \mathrm{~s}$ earlier on one side or the other. The SWD frequency ranged from 7.2 to $9.9 \mathrm{~Hz}$ (mean $8.1 \pm 0.5 \mathrm{~Hz}$ ) and the bursts, that lasted on average $18.9 \pm 9.1 \mathrm{~s}$, occurred irregularly at a frequency of about 50 to 150 per hour. Within the time elapsed from the first to the last recording session no change in periodicity of the SWD was detected. Quite often such SWD were followed by a low frequency pattern (2 to $4.8 \mathrm{~Hz}$ ) of discharges. While SWD occurred the animal remained immobilized but sometimes, and unexpectedly, clonic movements also accompanied the electrophysiological seizure.

Figure 1 shows three typical consecutive cortical SWD seizures similar in morphology and frequency $(9.5 \mathrm{~Hz})$ in area 10 of both brain hemispheres. Due to their high functional significance and relevance, these po- 
tentials will be described here in detail. In area 3 SWD oscillated at the same frequency on both sides but were different in morphology, although the electrodes were placed precisely over the contralateral symmetric site and their resistance was closely similar,

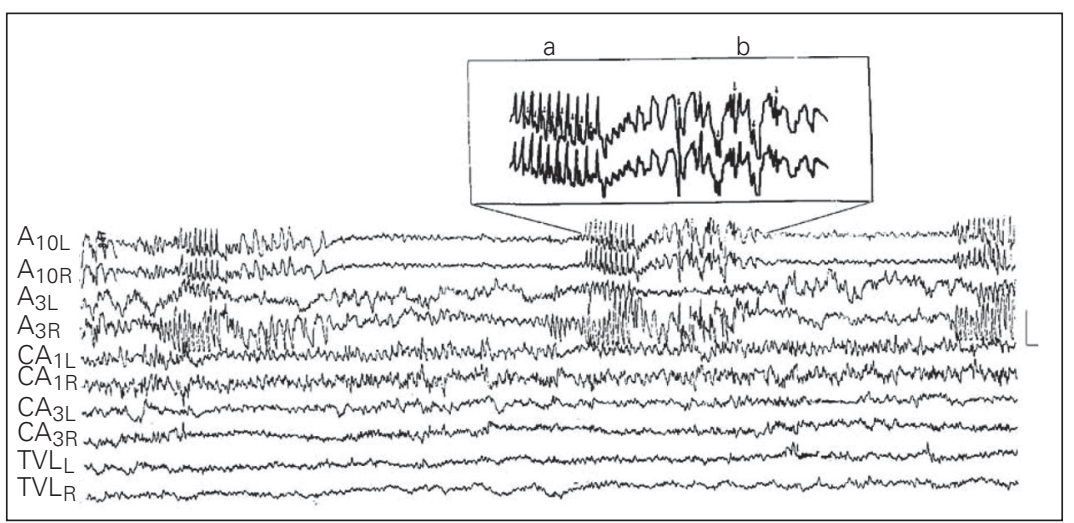

Figure 1. Bilateral bursts of spike-wave in neocortical areas 10 and 3 during an absence-like seizure. $A_{10}$ and $A_{3}$, areas 10 and 3 , respectively. $C A_{1}$ and $C A_{3}$, hippocampal fields 1 and 3 . $\mathrm{L}=$ left side; $\mathrm{R}=$ right side; $\mathrm{TVL}=$ thalamic ventral posterolateral nucleus. Inset, expanded

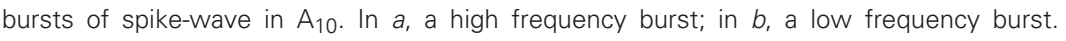
Calibration: $100 \mu \mathrm{V}, 1 \mathrm{~s}$.

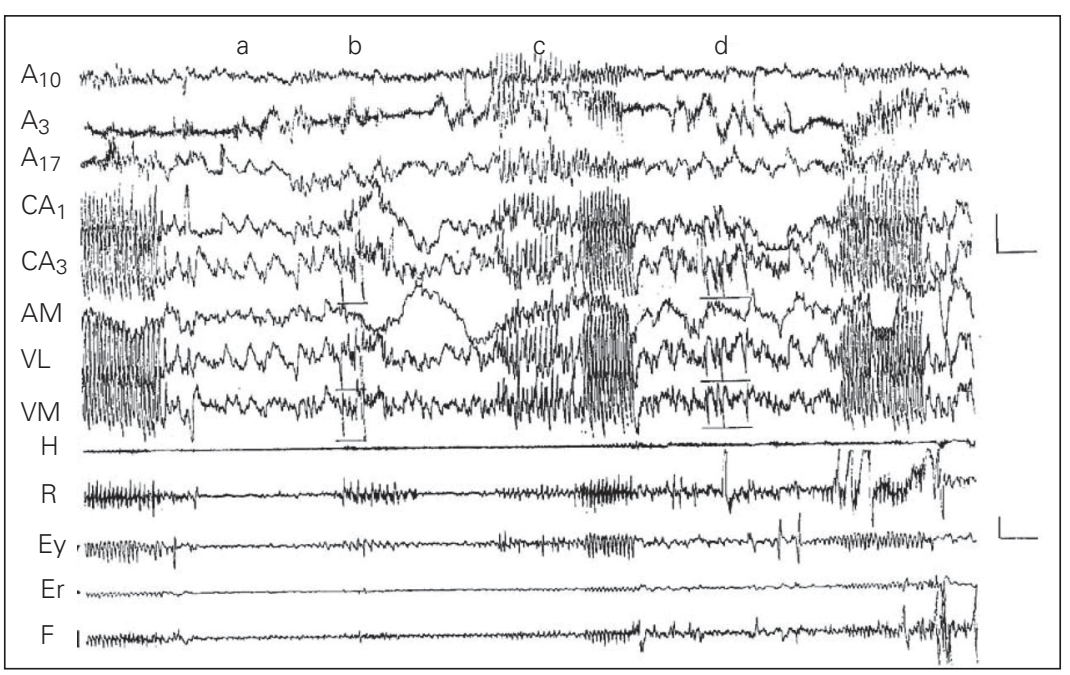

Figure 2. Three spike-wave discharge (SWD) bursts accompanied by clonic movements of the head $(H)$, rostrum + vibrissae $(R)$, eyes $(E y)$, ear $(E r)$ and left forelimb $(F)$. The frequency of the main spike-wave bursts is $9.5 \mathrm{~Hz}$. In a, a 2- $\mathrm{Hz}$ delta wave period follows the first spikewave burst, a frequent pattern after a seizure. Preceding the second burst (c) a lower frequency $(7 \mathrm{~Hz})$ discharge occurs that resembles the spike-wave burst. In $b$ and $d$, spike potentials that seem to be aborted SWD. The potentials in R, Ey, Er and F are not due to physical projection (artifacts) of the epileptogenic potentials in the brain. In $b$ the rostrum and eye movements lasted one second after the epileptogenic potentials. During the last spike-wave burst the eye and forelimb movements started before the epileptogenic potentials. $\mathrm{AM}=$ amygdala; $\mathrm{VL}$ and $\mathrm{VM}=$ ventrolateral and ventromedial nucleus of the hypothalamus. For other abbreviations, see legend to Figure 1. Calibration: $100 \mu \mathrm{V}, 1 \mathrm{~s}$. thus excluding the possibility of the difference being due to an artifact. Following the first and second SWD bursts, delta waves $(3.1 \mathrm{~Hz})$ occurred, coupled to a spike in the second burst, a pattern that was also a very common finding. As usual, the SWD complexes started as two or three slow waves not followed by spikes; then, the spikes started, closely linked to the delta waves, increased in voltage and disappeared before the slow waves, as is illustrated in Figure 1 (see inset). The inset shows with a higher definition that the earliest and the last potentials of a burst are only slow waves and that the slow wave and the spike component of the SWD burst are closely related ( $a$ and $b$ ). As will be discussed later, this is precisely the pattern for human absence seizure of the petit mal type.

The SWD were readily blocked by ethosuximide $(200 \mathrm{mg} / \mathrm{kg})$, an efficient antiepileptic drug. In 4 rats with recurrent SWD bursts in which we injected ethosuximide, the epileptic bursts were consistently and entirely blocked for $1 \mathrm{~h}$.

Most SWD neocortical bursts were accompanied by concomitant potentials in the hippocampus and other subcortical sites. In the same animal the cortical bursts may differ in voltage from the subcortical ones at different epochs. Figure 2 illustrates such correlations. In Figure 2a the low voltage cortical burst (in $A_{10}, A_{3}$ and $A_{17}$ ) is concomitant with a high voltage SWD burst in the hippocampal fields $\mathrm{CA}_{1}$ and $\mathrm{CA}_{3}$ as well as in the basolateral nucleus of the amygdala and in the ventromedial nucleus of the hypothalamus. The events in Figure $2 \mathrm{c}$ and d were equivalent in all leads. At the same time clonic movements occurred in several parts of the body, which were visually detectable during the seizure. In Figure $2 b$ repetitive spikes occur concomitantly in the subcortical structures, a pattern also observed for the seizures in Figure $2 \mathrm{c}$ and $\mathrm{d}$.

In Figure 3 the electro-oscillograms of an SWD seizure are reproduced from a com- 
puter-generated recording to increase the resolution of the potentials. In the right and left frontal areas 10 the spike-wave complexes are very clear but similar and synchronic complexes are present also in area $\mathrm{A}_{3}$ and in the hippocampus, mainly in the right $\mathrm{CA}_{1}$. The delta waves in the right left area 10, each followed by a spike, are in fact high voltage, low frequency SWD (with a saturated negative component). Even during an interval of 49 to $49.5 \mathrm{~s}$, when the SWD are absent from areas 10, they are quite clear in the right $\mathrm{CA}_{1}$.

In two rats, SWD were recorded during periods of paradoxical sleep. Figure 4a illustrates a normal episode of paradoxical sleep with rostrum and eye movements in one of the animals, characterized by desynchronization in area 10 and theta waves in area 3, in the hippocampal $\mathrm{CA}_{1}$ field and in the basolateral nucleus of the amygdala. The burst of SWD in Figure 4b appears suddenly in neocortical areas 10, 3 and 17 and in $\mathrm{CA}_{1}$ and is accompanied by phasic eye and ear movements. In Figure 4c, paradoxical sleep is again entirely normal.

In Figure 5 a short period of SWD with light clonic movements breaks through an episode of paradoxical sleep but, differing from the previous example, it is followed by wakefulness, during which desynchronization is present in area 3 , whereas in all the remaining leads theta waves occur. Concomitantly with the wakefulness electro-oscillogram, intense movements of the head, rostrum, eyes, ears and forelimb do appear with a normal aspect.

\section{Discussion}

The most significant findings of the present study are: i) permanent absence-like epilepsy develops in adult rats in which SE has been provoked by pilocarpine administration 7 to 17 days after birth; ii) iterative SWD occur bilaterally in the neocortex $(8.1 \pm 0.5$ $\mathrm{Hz}$ in frequency, $18.9 \pm 9.1 \mathrm{~s}$ in duration); iii)

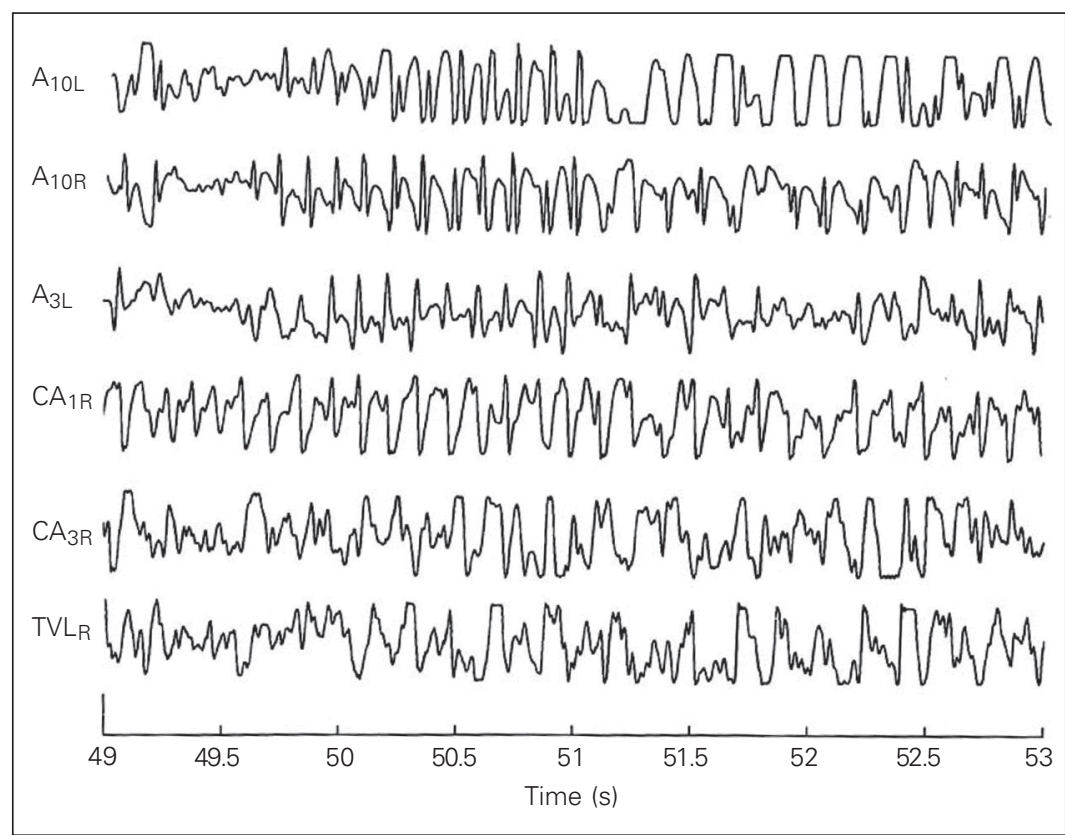

Figure 3. Tracings of an absence seizure recorded in a computer, demonstrating a perfect building up of a seizure spike-wave, which oscillates at $8 \mathrm{~Hz}$. Notice the concomitancy of the cortical and $\mathrm{CA}_{1}$ hippocampal potentials. In the latter, the slow waves are quite clear but the spikes are sometimes deformed, although clearly present. In $\mathrm{CA}_{3}$ and in the thalamic ventrolateral nucleus the slow potentials of the spike-wave complexes appear, although also deformed. Abscissa: time in 0.5-s intervals after the beginning of the acquisition period. For abbreviations, see legend to Figure 1.

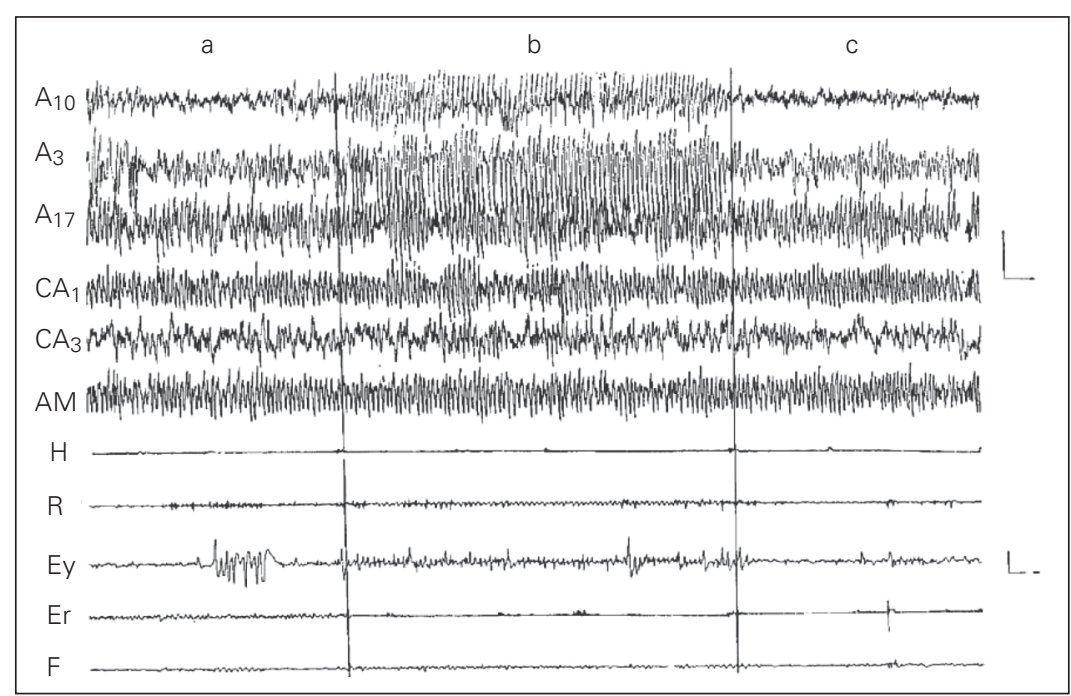

Figure 4. An episode of paradoxical sleep during which (b) a long (13 s) spike-wave burst occurred. In a and c normal periods of paradoxical sleep, with eye and rostrum movements and theta waves that characterize this phase of sleep. During the seizure, small rostrum and wider eye movements were present. Notice that in the basolateral nucleus of the amygdala theta waves are typical of desynchronized sleep, despite the seizure in neocortical and hippocampal sites. For abbreviations, see legends to Figures 1 and 2. Calibration: $100 \mu \mathrm{V}, 1 \mathrm{~s}$. 
during SWD the rats are generally immobilized, as in human absence seizures, but clonic movements and single jerks may occur concomitantly or dissociated from the SWD; iv) the neocortical SWD are similar to those found in all genetically epileptic rats; v) the SWD bursts can be entirely suppressed by ethosuximide; vi) similar SWD also always occur in the hippocampus and frequently appear in the basolateral nucleus of the amygdala, thalamic ventrolateral nucleus and hypothalamic ventromedial nucleus; vii) SWD may occur during paradoxical sleep.

Spike and wave complexes and immobilization are characteristic of absence fits in humans. Such seizures in humans may also be accompanied by jerks and clonic movements. Since our rats also showed such characteristics it is probable that their epilepsy is at least partially equivalent to that occurring in humans.

The pathognomonic electrophysiological signs of absence, or petit mal, epilepsy were studied in depth by several authors as soon as electroencephalography became available to neurologists. In humans, absence seizures

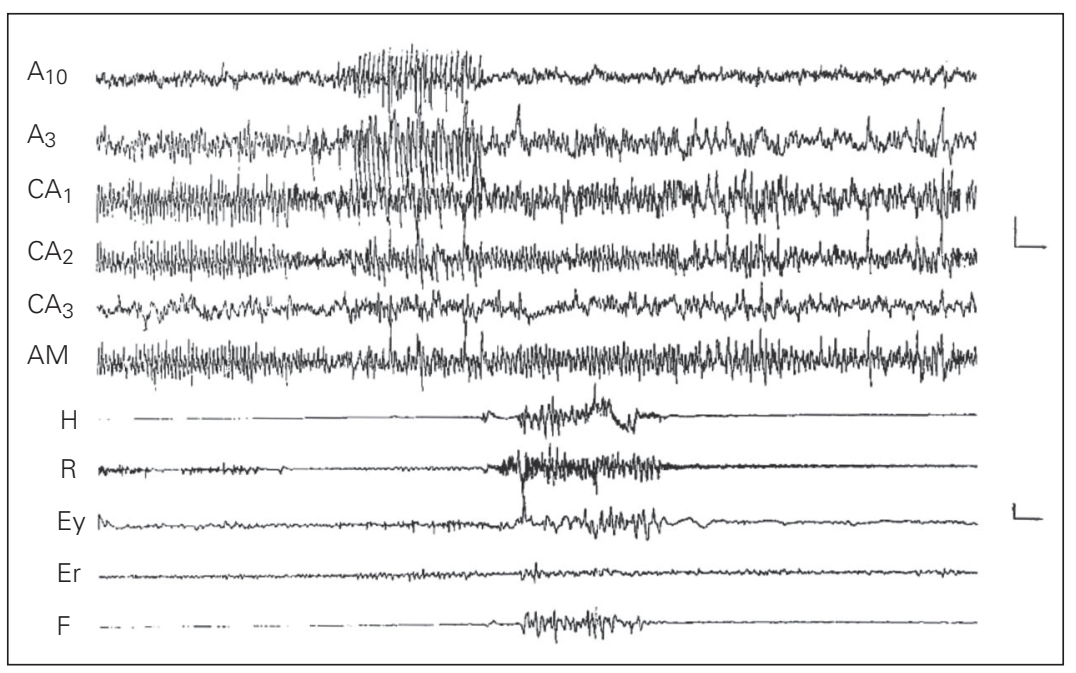

Figure 5. An episode of paradoxical sleep is followed by a burst of SWD and then a period of attentive wakefulness. During the paradoxical phase small rostrum and eye movements were present and during the wakefulness period theta waves and intense movements occurred. Notice that theta waves during the wakefulness period exhibit a lower voltage than during paradoxical sleep, as is the rule. For abbreviations, see legends to Figures 1 and 2. Calibration: $100 \mu \mathrm{V}, 1 \mathrm{~s}$. are electroencephalographically characterized by spike-wave complexes oscillating at nearly $3 \mathrm{~Hz}$ in both hemispheres, mainly in the frontal lobe. Recordings of potentials from both cortex and thalamic nuclei of six children with frequent absence fits (29) showed that the spike-wave complexes start in thalamic nuclei as delta waves that are transmitted to the cortex with increasing voltage as a function of time. Williams (29) advanced the hypothesis that the spike originates in deep cortical layers and propagates to the thalamic nuclei, with the consequent occurrence of a wave-spike pattern. After attaining a peak voltage, the SWD decreased in the opposite sequence, i.e., the spike diminished in voltage and eventually ceased while the delta wave was also progressively reduced in voltage and finally subsided. This pattern is similar to the cortical recruiting due to stimulation of midline and intralaminary nuclei (26), in agreement with the currently accepted hypothesis of a thalamic origin for SWD (30). It thus seems that the slow wave originates and progresses in the thalamus as a recruiting response and ascends to the cortex, where it also recruits the spike (29). Probably, the thalamically originated delta wave triggers the cortical spike, which in turn is projected back to thalamic nuclei; a resonant loop might thus arise and be responsible for the epileptic fit, which fails to occur when the spike is not recruited (29). The SWD we found in pilocarpine-treated rats evolved just as the human SWD in petit mal. Recent microelectrode studies have shown that the spikes of spike-wave complexes are associated with impulses leaving the cortex, thus supporting the hypothesis of the cortical origin of these potentials (29).

It should be pointed out, however, that the spike-wave complex may be triggered by penicillin $(31,32)$ in intact cats and by metrazol in the isolated cortex, which is suggestive of a specific cortical circuitry that is set to produce complex spike-wave complexes if adequately activated by subcortical 
discharges. Bilateral synchronous cortical spike-wave complexes have also been provoked by local application of kainic acid to the cingulate gyrus of cats (33), which introduces a complicating factor in the hypothesis of a thalamic origin of SWD.

The involvement of the hippocampus in absence seizures is of the utmost relevance for the understanding of its pathophysiology. However, this is still a controversial issue. In rodents, models of genetic absence seizures have been described $(34,35)$ in which spike-wave potentials were shown in cortical areas but not in the hippocampus. In the present study, in which epilepsy was provoked by treatment with pilocarpine, we have detected such discharges also in the hippocampus, as well as in the amygdala, thalamus and hypothalamus. This discrepancy may be attributed to the lesions provoked by the drug in different central structures, among which the hippocampus, amygdala and thalamus (21-24). It should be stressed, however, that in our rats the SWD in the hippocampus, amygdala and thalamus were not independent of the cortical ones but were concomitant, indicating that they are being driven by the same SWD pacemaker.

The question of SWD in the hippocampus has long been a matter of discussion. For instance, in stargazer mutant mice the study of the expression of neuropeptide $\mathrm{Y}$ in dentate gyrus granule cells (36) revealed that it is concentrated in mossy fibers following SWD seizures, a fact considered to reflect the gene induction triggered by synchronous bursting. The results of the present study clearly demonstrate that SWD are present in the hippocampus, which has been denied (33) due to the study of single/multi-unit recordings from the sensorimotor cortex, the ventrolateral thalamus and the hippocampal $\mathrm{CA}_{1}$ and $\mathrm{CA}_{3}$ fields. Whereas these recordings demonstrated a tight correlation between the unit recordings and the spike component of the SWD in the cortex and thalamus, no correlation was detected in the hip- pocampus. Another demonstration of the lack of hippocampal activation during absence seizures induced by GABA is the expression of $\alpha 1$ and $\alpha 4$ subunit mRNA in thalamic relay nuclei but not in the hippocampus (37). Patterns of identifiable spike-wave components were recorded from the human hippocampus (38) during temporal lobe epilepsy but such discharges were taken as the effect of rhythmic patterns generated within the hippocampus but without any relation to SWD epilepsy (39).

In kainate-treated rats, a protocol that induces epilepsy, $\mathrm{CA}_{1}$ pyramidal cells and putative inhibitory neurons in the stratum oriens were reduced within eight days after kainate administration and axon branching of $\mathrm{CA}_{1}$ pyramidal cells was increased several weeks after kainate treatment (24). In rats submitted to pilocarpine administration interneurons in $\mathrm{CA}_{1}$, amygdala and pyriform cortex have been shown to be primarily lesioned; repeated seizures seem to increase the number of inhibitory interneurons that are destroyed, thus facilitating the pathological excitation that results in epileptic seizures, and pilocarpine also damages cortical neurons one day after being injected in rats (24). It is very interesting and relevant that when domoic acid is injected in normal rats, it causes immediate SE, which may last up to $24 \mathrm{~h}$, but if injected in spontaneously epileptic rats $\mathrm{SE}$ is either short-lasting or even absent (40).

In the laboratory of one of us, a strain of rats (FMUSP-rats) with spontaneous absencelike seizures was found $(41,42)$, in which SWD are profuse in the hippocampus and other subcortical nuclei. In these rats the absence epilepsy is spontaneous (probably genetic) and thus does not involve a druginduced lesion of the hippocampus. The occurrence of SWD in the FMUSP-rats is not compatible with the statement that in the hippocampus such discharges never occur in spontaneously epileptic rats. It is interesting that, despite the lack of electrophysiological 
epileptogenic potentials in the hippocampus of another genetically epileptic absence strain of rats, a marked increase in local glucose utilization in the dorsal hippocampus $(+36 \%)$, in the ventral hippocampus $(+37 \%)$ and in the dentate gyrus has been consistently observed, in addition to increased metabolism in several other central structures (43).

The data cited above reveal that there is a serious discrepancy among several studies regarding the presence of SWD in the hippocampus. Several researchers adhered to the dogmatic conclusion that the hippocampus is not involved in the so-called absence models, whereas a few investigators disagree with this position. Unless some experiments have not been correctly performed, which is unlikely, it should be accepted that in some species or strains the hippocampus is involved in the production of SWD and absence seizures, whereas in others it is not.

Lesioning induced by pilocarpine is only partially understood. It does probably involve many regions of the central nervous system that have not yet been investigated, a fact that limits reasoning about which central areas are in fact affected and how some functional epileptic manifestations can be explained. For instance, pilocarpine-induced SE in early life significantly changes the predominance of movements exhibited during paradoxical sleep (20). Eye movements, which in normal rats occur only during $13 \%$ of the time, last $42.8 \%$ of the time in pilocarpine-induced epilepsy, whereas rostrum movements, which occur during $48 \%$ of the time in normal rats (Valle AC and TimoIaria $\mathrm{C}$, unpublished results), decrease to only $8.1 \%$ in the epileptic animals (20). Since paradoxical sleep depends on pontine structures, impairment of such structures is likely to occur in epileptic rats.

Jerks do occur in about $30 \%$ of children with absence attacks (2), which in some patients arise from a diffuse epileptic encephalopathy, such as Lennox-Gastaut syndrome. Thus, it is likely that the seizures provoked by pilocarpine mimic this syndrome, since absence attacks and jerks and clonic movements did occur consistently in the rats studied here.

Absence seizures have been considered to be generated in thalamic circuits, possibly arising from aberrant thalamocortical circuits (30), a hypothesis that is consistent with the centrencephalic model proposed by Penfield and Jasper (44) to explain the loss of consciousness during epileptic seizures. It is remarkable that, although their morphology is very similar, the frequency of oscillation of the spike-wave complexes in humans (about $3 \mathrm{~Hz}$ ) and rodents (about $8 \mathrm{~Hz}$ ) is quite different. If the hypothesis that generalized absence seizures are a perturbation of neocortical rhythmicity in favor of the rhythmic burst firing mode is correct, this difference may be attributed to the specificity of frequency patterns in the nervous system of organisms occupying different phylogenetic positions. A good example of such specificity is that whereas desynchronization is characteristic of arousal and desynchronized (paradoxical) sleep in most cortical areas in man and other primates, in rats and other rodents it is present almost exclusively in the frontal cortex, while theta waves characterize both states of the wakefulness-sleep cycle (45). It is certainly not a coincidence that SWD occur within the frequency domain of theta waves $(7$ to $10 \mathrm{~Hz}$ ). Probably, the oscillatory frequency of theta waves is related to SWD frequency because some circuits that give origin to both phenomena are the same or are influenced by circuits in which theta waves are present.

The prompt and enduring effect of ethosuximide administration in blocking the SWD in pilocarpine-induced seizures points to a high similarity between such fits in our rats and in absence seizures of genetic origin.

A relevant issue in the present study is that in two rats SWD occurred during episodes of paradoxical sleep. Epileptic absence is clearly related to interruption of the 
conscious process, and dreaming activity, which is a conscious activity, occurs during paradoxical sleep. This is a very interesting and relevant phenomenon that remains to be studied in depth because it may bring information about consciousness itself. Petit mal seizures are well characterized by the sudden block of consciousness and total arrest of responses to sensory stimulation. If such a drastic effect is concomitant with a real absence of mental activity is not known. It may be only an interruption of the connection between the sensory channels and the conscious processing of information, or the absence of memory consolidation, which does not allow the patient to recall anything that occurred during the seizure. If this is the case, the occurrence of dreaming activity in rats during the epileptic seizure, as we found here, is not surprising. Anyhow, this interesting problem requires further advanced experimental investigation.

\section{References}

1. Gherpelli JLD, Paz JA, Leone CR, Ramos JLA \& Diament A (1992). Seizure recurrence in infants with neonatal convulsions. Arquivos de Neuro-Psiquiatria, 50: 31-36.

2. Hauser WA (1994). The prevalence and incidence of convulsive disorders in children. Epilepsia, 35 (Suppl 2): S1-S6.

3. Aicardi J \& Chevie JJ (1983). Consequences of status epilepticus in infants and in children. In: Delgado-Escueta AV, Wasterlein CG, Treiman DM \& Porter RJ (Editors), Advances in Neurology, Status Epilepticus: Mechanisms of Brain Damage and Treatment. Raven Press, New York.

4. Lombroso CT (1977). Convulsive disorders in newborns. In: Thompson RS \& Green JR (Editors), Pediatric Neurology and Neurosurgery. Spectrum Publications, New York.

5. Aminoff MJ \& Simon RP (1980). Status epilepticus: causes, clinical features and consequences in 98 patients. American Journal of Medicine, 69: 659-666.

6. Maytal J, Shinnar S, Moshé SL \& Alvarez LA (1989). Low morbidity and mortality of status epilepticus in children. Pediatrics, 83: 323331.

7. Mitchell WG (1996). Status epilepticus and acute repetitive seizures in children, adolescents, and young adults: etiology, outcome and treatment. Epilepsia, 37 (Suppl. 1): S74-S80.

8. Corselis JAN \& Bruton CJ (1983). Neuropathology of status epilepticus in humans. In: Delgado-Escueta AV, Wasterlein CG, Treiman DM \& Porter RJ (Editors), Advances in Neurology. Status Epilepticus: Mechanisms of Brain Damage and Treatment. Raven Press, New York.

9. Ben-Ari Y (1985). Limbic seizure and brain damage produced by kainic acid: mechanisms and relevance to human temporal lobe epilepsy. Neuroscience, 14 (Suppl 2): 375-403.

10. Cavalheiro EA, Silva DF, Turski WA, Calderazzo-Filho LS, Bortolotto ZA \& Turski L (1987). The susceptibility of rats to pilocarpineinduced seizures is age-dependent. Brain Research, 465: 43-58.

11. Cavalheiro E, Santos NF \& Priel MR (1996). The pilocarpine model of epilepsy in mice. Epilepsia, 37: 1015-1019.

12. Chapman A, Ingmar M \& Siesjo BK (1980). Free fatty acids in the brain in bicuculline-induced status epilepticus. Acta Physiologica Scandinavica, 110: 335-336.

13. Leite JP, Bortolotto ZA \& Cavalheiro EA (1990). Spontaneous recurrent seizures in rats: an experimental model of epilepsy. Neuroscience and Biobehavioral Reviews, 14: 511-517.

14. Lemos T \& Cavalheiro EA (1995). Suppression of pilocarpine-in- duced status epilepticus and the development of epilepsy in rats Experimental Brain Research, 102: 423-428.

15. Albala BJ, Moshé SL \& Okada R (1984). Kainic acid-induced seizures: a developmental study. Brain Research, 315: 139-148.

16. Kellaway $P$ (1982). Maturation and biorhythmic changes in the electroencephalogram. In: Anderson VE, Hauser WA \& Penny JK (Editors), Genetic Basis of the Epilepsies. Raven Press, New York.

17. Ounstead C (1971). Some aspects of seizure disorders. In: Gardner D \& Hull D (Editors), Recent Advances in Pediatrics. Churchill, London, England.

18. Priel MR, Santos NF \& Cavalheiro EA (1996). Developmental aspects of the pilocarpine model of epilepsy. Epilepsy Research, 26: 115-121.

19. Ferreira BLC, Bortolotto ZA \& Cavalheiro EA (1995). Anticonvulsant drugs and pilocarpine-induced status epilepticus. Epilepsia, 36: S48 (Abstract).

20. Ferreira BLC, Valle AC, Cavalheiro EA \& Timo-laria C (1999). Prevalence of epileptic seizures along the wakefulness-sleep cycle in rats submitted to status epilepticus in early life. Developmental Neuroscience, 21: 339-344.

21. Mello LEAM \& Covolan L (1996). Spontaneous seizures preferentially injure interneurons in the pilocarpine model of chronic spontaneous seizures. Epilepsy Research, 26: 123-129.

22. Covolan L, Ribeiro LTC, Longo BM \& Mello LEAM (2000). Cell damage and neurogenesis in the dentate granule cell layer of adult rats after pilocarpine- or kainate-induced status epilepticus. Hippocampus, 10: 169-180.

23. Covolan L, Smith RL \& Mello LEAM (2000). Ultrastructural identification of dentate granule cell death from pilocarpine-induced seizures. Epilepsy Research, 41: 9-21.

24. Covolan L \& Mello LEAM (2000). Temporal profile of neuronal injury following pilocarpine or kainic acid-induced status epilepticus. Epilepsy Research, 39: 133-152.

25. Timo-laria C, Negrão N, Schmidek WR, Rocha TL \& Hoshino K (1970). Phases and states of sleep in the rat. Physiology and Behavior, 5: 1057-1062.

26. Timo-laria C, Negrão N \& Pereira WC (1971). Spindling during natural and barbiturate sleep, in the isolated cerebrum and in recruiting in the rat brain. In: Kao FF, Koizumi K \& Vassale M (Editors), Research in Physiology, A. Gaggi Publisher, Bologna, Italy.

27. Zilles K (1991). Anatomy of the neocortex, cytoarchitecture and myeloarchitecture. In: Kolb B \& Tess RC (Editors), The Cerebral 
Cortex of the Rat. The MIT Press, Cambridge, MA, USA.

28. Paxinos G \& Watson C (1997). The Rat Brain in Stereotaxic Coordinates. Academic Press, Sydney, Australia.

29. Williams $D$ (1953). A study of thalamic and cortical rhythms in petit mal. Brain, 76: 50-69.

30. Steriade M (1990). Spindling, incremental thalamocortical responses, and spike-wave epilepsy. In: Avboli M, Gloor P, Kospoulos $G$ \& Naquet $T$ (Editors), Generalized Epilepsy. Birkauser, Boston, MA, USA.

31. Fisher RS \& Prince DA (1977). Spike-wave rhythm in cat cortex induced by parenteral penicillin. II. Cellular features. Electroencephalography and Clinical Neurophysiology, 42: 65-39.

32. Gloor P (1968). Generalized cortico-reticular epilepsies. Some considerations on the pathophysiology of generalized bilaterally synchronous spike and wave discharge. Epilepsia, 9: 249-263.

33. Cukiert A (1996). A role for cortex and corpus callosum in physiopathology of the secondary bilateral synchronous discharges in the cat. Doctoral thesis, University of São Paulo, São Paulo, SP, Brazil.

34. Marescaux C, Vergnes M \& Depaulis A (1992). Genetic absence epilepsy in rats from Strasbourg. A review. Journal of Neural Transmission, 35: 37-69.

35. Coenen $A M$, Drinkenburg $W H$, Inoue $M$ \& van Luijtelaar ELJM (1992). Genetic models of absence epilepsy, with emphasis on the WAG/Rij strain of rats. Epilepsy Research, 12: 75-86.

36. Chafetz RS, Nahm WK \& Noebels JL (1995). Aberrant expression of neuropeptide $Y$ in hippocampal mossy fibers in the absence of local cell injury following the onset of spike-wave synchronization. Molecular Brain Research, 31: 111-121.
37 Banerjee PK, Tillakaratne NJK, Brailowski S, Olsen RW, Tobin AJ \& Snead III OC (1998). Alterations in $\mathrm{GABA}_{\mathrm{A}}$ receptor $\alpha 1$ and $\alpha 4$ subunit mRNA levels in thalamic relay nuclei following absence-like seizures in rats. Experimental Neurology, 154: 213-223.

38. Engel Jr J (1989). Seizures and Epilepsy. F.A. Davis, Philadelphia, PA, USA.

39. Kandel A, Bragin A, Carpi D \& Buszáki G (1996). Lack of hippocampal involvement in a rat model of petit mal epilepsy. Epilepsy Research, 23: 123-127.

40. Alves RSC (1999). Status epilepticus in rats induced by domoic acid. Doctoral thesis. School of Medicine, University of São Paulo, São Paulo, SP, Brazil.

41. Valle AC, Ferreira BLC, Alves RSC \& Timo-laria C (1998). Spontaneous spike-wave discharges in adult rats. Epilepsia, 39 (Suppl): 26 (Abstract).

42. André ES, Bruno-Neto R, Valle AC \& Timo-laria C (1999). Electrophysiological characterization of a new form of spontaneous epilepsy in Wistar rats. Epilepsia, 42 (Suppl): 40 (Abstract).

43. Nehlig A, Vergnes M, Marescaux C, Boyet S \& Lannes B (1991). Local cerebral glucose utilization in rats with petit mal-like seizures. Annals of Neurology, 29: 72-77.

44. Penfield W \& Jasper H (1954). Epilepsy and the Functional Anatomy of the Human Brain. Little, Brown \& Company, Boston, MA, USA.

45. Valle AC, Timo-laria C, Sameshima K \& Yamashita R (1992) Theta waves and behavioral manifestations of alertness and dreaming activity in the rat. Brazilian Journal of Medical and Biological Research, 25: 745-750. 